\title{
Comparative Analysis of the Biomechanical Characteristics After Different Minimally Invasive Surgeries for Cervical Spondylopathy: A Finite Element Analysis
}

Tao He, Jun Zhang, Tong Yu, Jiuping Wu, Tianyang Yuan, Rui Liu, Zhihe Yun, Haorui Du, Le Qi, Junyan An, Wu Xue, Xinyu Nie and Qinyi Liu*

Department of Spine Surgery, The Second Hospital of Jilin University, Jilin University, Changchun, China

OPEN ACCESS

Edited by:

Alexandros E. Tsouknidas,

University of Western Macedonia,

Greece

Reviewed by:

Wenbin Hua,

Huazhong University of Science and

Technology, China

Panagiotis Chatzistergos,

Staffordshire University,

United Kingdom

*Correspondence:

Qinyi Liu

qinyi@jlu.edu.cn

Specialty section:

This article was submitted to

Biomechanics,

a section of the journal

Frontiers in Bioengineering and

Biotechnology

Received: 08 September 2021

Accepted: 22 November 2021

Published: 16 December 2021

Citation:

He T, Zhang J, Yu T, Wu J, Yuan T,

Liu R, Yun Z, Du H, Qi L, An J, Xue W,

Nie $X$ and Liu Q (2021) Comparative

Analysis of the Biomechanical

Characteristics After Different Minimally

Invasive Surgeries for Cervical

Spondylopathy: A Finite

Element Analysis.

Front. Bioeng. Biotechnol. 9:772853.

doi: 10.3389/fbioe.2021.772853
Minimally invasive surgeries, including posterior endoscopic cervical foraminotomy (PECF), microsurgical anterior cervical foraminotomy (MACF), anterior transdiscal approach of endoscopic cervical discectomy (ATd-ECD), and anterior transcorporeal approach of endoscopic cervical discectomy (ATc-ECD), have obtained positive results for cervical spondylotic radiculopathy. Nonetheless, there is a lack of comparison among them regarding their biomechanical performance. The purpose of this study is to investigate the biomechanical changes of operated and adjacent segments after minimally invasive surgeries compared to a normal cervical spine. A three-dimensional model of normal cervical vertebrae C3-C7 was established using finite element analysis. Afterwards, four surgical models (PECF, MACF, ATd-ECD, and ATC-ECD) were constructed on the basis of the normal model. Identical load conditions were applied to simulate flexion, extension, lateral bending, and axial rotation of the cervical spine. We calculated the range of motion (ROM), intradiscal pressure (IDP), annulus fibrosus pressure (AFP), uncovertebral joints contact pressure (CPRESS), and facet joints CPRESS under different motions. For all circumstances, ATc-ECD was close to the normal cervical spine model, whereas ATdECD significantly increased ROM and joints CPRESS and decreased IDP in the operated segment. PECF increased more the operated segment ROM than did the MACF, but the MACF obtained maximum IDP and AFP. Except for ATc-ECD, the other models increased joints CPRESS of the operated segment. For adjacent segments, ROM, IDP, and joints CPRESS showed a downward trend in all models. All models showed good biomechanical stability. With their combination biomechanics, safety, and conditions of application, PECF and ATC-ECD could be appropriate choices for cervical spondylotic radiculopathy.

\footnotetext{
Keywords: anterior transdiscal approach of endoscopic cervical discectomy, posterior endoscopic cervical foraminotomy, microsurgical anterior cervical foraminotomy, anterior transcorporeal approach of endoscopic cervical discectomy, cervical minimally invasive surgery, biomechanics, finite element analysis
}

Abbreviations: AFP, annulus fibrosus pressure; ATd-ECD, anterior transdiscal approach of endoscopic cervical discectomy; ATc-ECD, anterior transcorporeal approach of endoscopic cervical discectomy; CPRESS, contact pressure; CT, computed tomography; EXT, extension; FLE, flexion; FJs, facet joints; IDP, intradiscal pressure; LAR, left axial rotation; LLB, left lateral bending; MACF, microsurgical anterior cervical foraminotomy; PECF, posterior endoscopic cervical foraminotomy; RAR, right axial rotation; RLB, right lateral bending; ROM, rang of motion; UJs, uncovertebral joints. 


\section{INTRODUCTION}

Cervical spondylotic radiculopathy is usually characterized by pain and numbness of the neck, shoulders, and arms as well as restriction of cervical movement, which significantly decreases quality of life for patients (Yuchi et al., 2019; Chen et al., 2020). Anterior cervical discectomy and fusion has turned into a standard procedure for cervical spondylotic radiculopathy because of its safety, effectiveness, and high fusion rate since the 1950s (Ruetten et al., 2009; Yang et al., 2014; Ren et al., 2019), notwithstanding that it may cause some problems such as degeneration of adjacent segments, loss of intervertebral disc height, and pseudarthrosis formation (Ruetten et al., 2009; Yang et al., 2014; Yuchi et al., 2019; Chen et al., 2020). With the development of full-endoscopic cervical discectomy, the complications of anterior cervical discectomy and fusion were managed appropriately (Ruetten et al., 2009; Wu et al., 2018; Ahn, 2020). Full-endoscopic cervical discectomy is generally divided into two types, i.e., anterior transdiscal approach of endoscopic cervical discectomy (ATd-ECD) and posterior endoscopic cervical foraminotomy (PECF) (Yang et al., 2014; Ren et al., 2020). PECF is an indirect decompression of the technique through the posterior approach, which requires the removal of the partial bony structure and soft tissue with a radius of 3-4 mm around the $\mathrm{V}$-point (inferior margin of the cephalic lamina, superior margin of the caudal lamina, and the medial border of facet joints [FJ]) to decompress the nerve root (Kim et al., 2015; Ahn, 2016; Won et al., 2017; Wu P. H. et al., 2021). The ATd-ECD technique can achieve precision and direction of approach using contrast agents, but a tunnel needs to be built in the intervertebral disc; after that, the endoscopic instruments remove the protruded disc through the intervertebral space (Lee and Lee, 2014; QuilloOlvera et al., 2018; Haijun et al., 2021). Nowadays, PECF and ATd-ECD have gradually become alternative options for spine surgeons in treatment of cervical disc herniation because of their good postoperative stability and high clinical success rate (Ruetten et al., 2008; Yang et al., 2014).

ATd-ECD generates greater iatrogenic disc injury and causes intervertebral space decrease (Ren et al., 2020). With regard to better disc preservation, a similar technique named anterior transcorporeal approach of endoscopic cervical discectomy (ATc-ECD) was used subsequently (Choi et al., 2007). ATcECD can relieve the compression of the nerve root by drilling a hole in the vertebral body, which avoids the unnecessary destruction of the intervertebral disc and bony stabilizers because it can reach the region of the protruded disc or uncovertebral osteophyte through the bone tunnel (Choi et al., 2007; Kim et al., 2011; Umebayashi et al., 2013; Deng et al., 2016; Wu et al., 2018; Chen et al., 2021). Ren et al. (2020) reported that the rate of excellent or good results reached up to $91.4 \%$, and intervertebral space decrease was reported after ATc-ECD. However, it was not revealed whether intervertebral space decrease after ATc-ECD causes the apparent biomechanical changes of structures, like the intervertebral disc, FJs, and uncovertebral joints (UJs). Previous experiments focused primarily on vertebral strength change after operation
(Umebayashi et al., 2013; Ren et al., 2020); thus, the biomechanics of ATc-ECD need to be further explored in detail.

Aside from a herniated disc, secondary osseous foraminal stenosis resulting from UJ osteophytes can also potentially induce cervical spondylotic radiculopathy (Boreadis and GershonCohen, 1956; Snyder et al., 2007; Brismée et al., 2009; Clifton et al., 2020). Previous research demonstrated that the direct decompression of foraminal stenosis by resecting UJ effectively relieved the symptoms of nerve compression (Jho, 1997; Saringer et al., 2002; Saringer et al., 2003; Kotil and Bilge, 2008). MACF is regarded as an alternative functional surgery and can preserve segmental motion compared with anterior cervical discectomy and fusion (Maduri et al., 2020). And it can also represent a supplement surgery to better improve arm pain after conducting anterior cervical discectomy and fusion (Lee et al., 2018; Clifton et al., 2020; Noh et al., 2020). A key step of MACF is the necessary removal of hypertrophied UJs to expand the intervertebral foramen and decompress the nerve root (Taşçioğlu et al., 2001; Clifton et al., 2019). However, UJs are regarded as a stabilizer to limit cervical posterior translation and lateral bending, and resection of UJs will disrupt stability and may add a load on other bearing structures (Kotani et al., 1998; Wang et al., 2016). Kotani et al. (1998) divided UJs into three parts, namely, the posterior foraminal part, the posterior half, and the anterior half, and separately analyzed the stability of each part; however, the detailed biomechanical responses inside of the cervical spine were not exposed.

Previous studies showed that PECF obtained better postoperative stability than did ATd-ECD (Yuchi et al., 2019; Chen et al., 2020). Still, there is a lack of comparative study of biomechanics for the four aforementioned minimally invasive surgeries, and it is unclear which surgery represents optimal stability. Besides, the variations of UJs have not been discussed after cervical surgeries. Due to limitations of technology, it is currently impossible to conduct biomechanical in vivo experiments; additionally, in vitro experiments are not easy to carry out, because specimens are expensive and unavailable (Ames et al., 2005; Yuchi et al., 2019). Finite element analysis can deal with these limitations and obtain satisfactory results as well as detailed internal information of the cervical spine; furthermore, it can possibly provide interpretations for postoperative symptoms (Yuchi et al., 2019). As a digital research tool, finite element analysis can assess cervical spine kinematics or biomechanics, can simulate various clinical situations, and has been widely applied to spine biomechanical research so far (Wu W. et al., 2021; Sang et al., 2021).

In our study, we analyze the biomechanical characteristics after performing different minimally invasive surgeries in light of range of motion of segments and the pressure on the following structures: intervertebral disc, FJs, and UJs. The objective of this study is to assess and compare the cervical biomechanical performance of the four surgical models (PECF, MACF, ATd$\mathrm{ECD}$, and ATc-ECD) against the preoperative three-dimensional finite element model of normal cervical vertebrae C3-C7. After analyzing and comparing the changes of ROM, AFP, IDP, FJs, and UJs CPRESS between preoperation and postoperation, we 

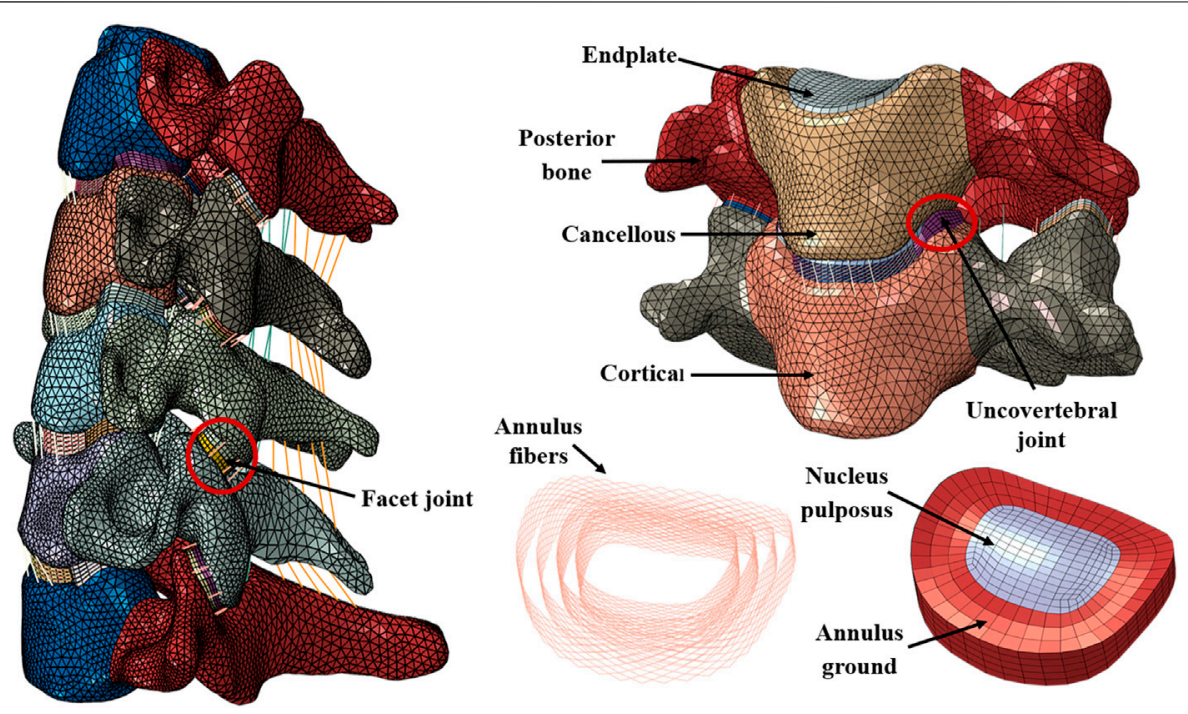

FIGURE 1 | Finite element model of intact $\mathrm{C} 3-\mathrm{C} 7$ and components.

expect to provide useful information for spine surgeons regarding the selection of operation plans.

\section{MATERIALS AND METHODS}

\section{Establishment of Three-Dimensional Model}

The data of the C3-C7 finite element model establishment stemmed from a computed tomography (CT) scan of a healthy subject (gender: male; age: 25; height: $176 \mathrm{~cm}$; weight: $65 \mathrm{~kg}$ ). The subject does not have any symptoms of cervical spondylopathy and feelings of neck discomfort. The conversion of CT data to STL files was conducted in Mimics 21.0 (Materialise Inc., Leuven, Belgium). Then, the STL files were imported into the Geomagic Wrap 2017 software (Geomagic, Inc., Research Triangle Park, NC, United States) for smoothing the original model. According to the anatomical position and shape of disc, UJs, and FJs, we used the SOLIDWORKS 2018 (Dassault Systèmes Inc., France) software to generate them step by step. The construction of the mesh model and the finite element preprocessing were done using HyperMesh 14.0 (Altair Engineering, Inc., Executive Park, CA, United States). Finally, the finite element model calculation and analysis were implemented with the Abaqus 2020 software (Abaqus, Inc., Providence, RI, United States). The intact model and its components are presented in Figure 1.

The intact finite element model consisted of the cortical bone, cancellous bone, posterior bone, endplate, annulus ground, annulus fibers, nucleus pulposus, FJ, UJ, and five major ligaments. The endplate was located between the intervertebral disc and cancellous bone. The cortical bone and endplate were assumed as $0.5 \mathrm{~mm}$ thin bony shells. The nucleus pulposus was defined as a non-compressible material, and the volume rate between nucleus pulposus and annulus ground was 4:6 (Wu et al.,
2019; Hua et al., 2020; Sang et al., 2021). The annulus fibers accounted for approximately $20 \%$ of ground volume, and they were circumferential around the surface of the annulus ground at an angle of approximately $15^{\circ}-45^{\circ}$ with respect to the endplate horizontal plane (Mo et al., 2015). Based on the mesh model, five ligaments, i.e., anterior longitudinal ligament, posterior longitudinal ligament, capsular ligament, interspinous ligament, and ligamentum flavum, were constructed node to node.

Facet cartilages were split into two equal inferior and superior parts. The contact method between facet cartilages was modeled as frictional contact, with the surface-to-surface method. Additionally, the friction coefficient was 0.1 (Wong et al., 2020; Wo et al., 2021). The contact between UJs and cortical bone was identical to facet cartilages. For convenience reasons, we assumed the UJs as hexahedrons and neglected its anatomical fissure.

\section{Material Properties and Element Types}

The intact finite element model comprised 254,406 elements and 67,194 nodes. Fibers and ligaments were simulated by tensiononly truss elements. The vertebral body was simulated by fournode tetrahedral elements, and the rest of the material was simulated by eight-node hexahedral elements. The detailed material properties (Lee et al., 2011; Cai et al., 2020a; Guan et al., 2020; Hua et al., 2020; Ke et al., 2020; Wang et al., 2020; Sang et al., 2021; Wo et al., 2021) of all parts are presented in Table 1.

\section{Construction of Postoperative Models}

All postoperative models were altered on the basis of the intact model. The four postoperative models are shown in Figure 2. A tunnel with a $3.9 \mathrm{~mm}$ diameter was established on the C5-C6 intervertebral disc from the right front to the left rear based on the 
TABLE 1 | The mechanical property of the components of the C3-C7 finite element model.

\begin{tabular}{|c|c|c|c|c|}
\hline Components & Young's modulus (MPa) & Poisson's ratio & Element type & Cross-sectional area $\left(\mathrm{mm}^{2}\right)$ \\
\hline Cortical bone & 12,000 & 0.3 & C3D4 & - \\
\hline Cancellous bone & 450 & 0.29 & C3D4 & - \\
\hline Posterior bone & 3,500 & 0.29 & C3D4 & - \\
\hline Endplate & 500 & 0.4 & C3D8R & - \\
\hline Annulus ground & 3.4 & 0.4 & $\mathrm{C} 3 \mathrm{D} 8 \mathrm{H}$ & - \\
\hline Annulus fibers & 110 & 0.3 & Tension-only truss & - \\
\hline Nucleus pulposus & 1 & 0.49 & $\mathrm{C} 3 \mathrm{D} 8 \mathrm{H}$ & - \\
\hline Facet joints & 10 & 0.4 & C3D8R & - \\
\hline Uncovertebral joints & 10 & 0.4 & C3D8R & - \\
\hline Anterior longitudinal ligament & 10 & 0.3 & Tension-only truss & 6 \\
\hline Posterior longitudinal ligament & 10 & 0.3 & Tension-only truss & 5 \\
\hline Capsular ligament & 10 & 0.3 & Tension-only truss & 46.6 \\
\hline Ligament flavum & 1.5 & 0.3 & Tension-only truss & 5 \\
\hline Interspinous ligament & 1.5 & 0.3 & Tension-only truss & 10 \\
\hline
\end{tabular}
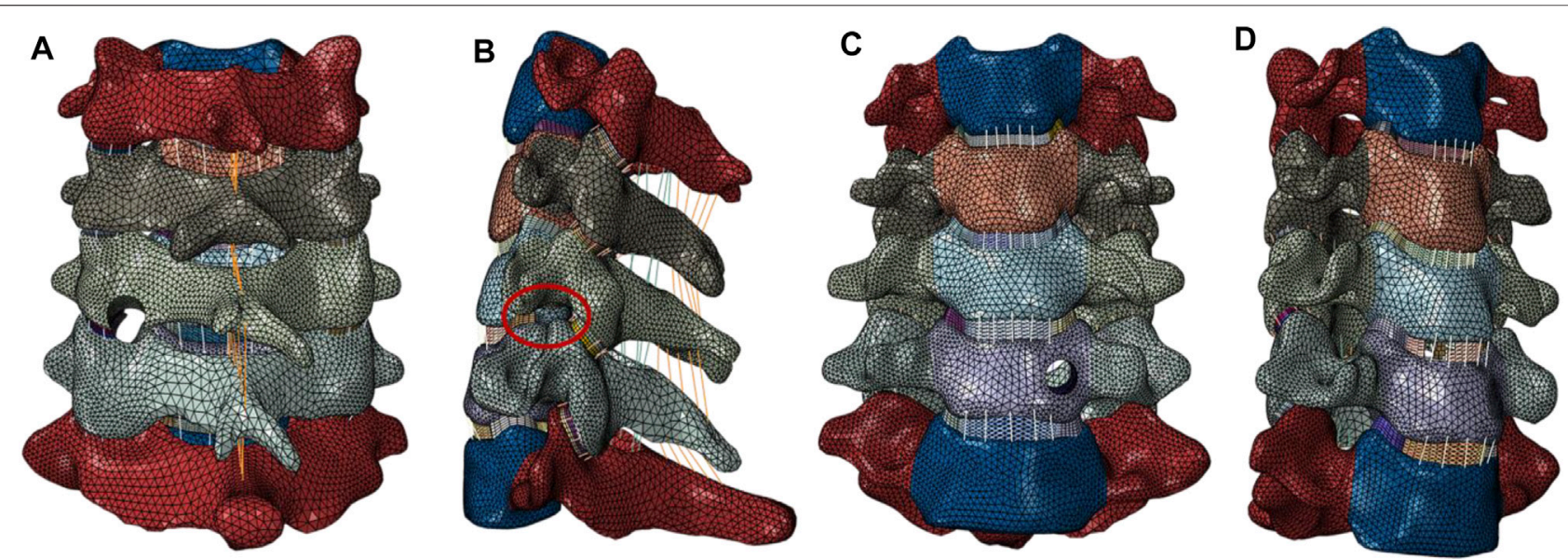

FIGURE 2 | (A) PECF: a $7 \mathrm{~mm}$ diameter tunnel was constructed around the V-point at the C5-C6 left FJ; and partial articular capsular and laminae were removed. (B) MACF: the posterior part of the left UJ was removed at the C5-C6 segment without disc resection. (C) ATc-ECD: a 6 mm diameter tunnel was designed on the C6 vertebral body left side from anterior inferior towards posterior superior, and endoscopic instruments can reach the region of the compressed nerve root. (D) ATd-ECD: a $3.9 \mathrm{~mm}$ diameter tunnel was constructed at the C5-C6 intervertebral space from the right front to the left rear, and the target area was the left lateral recess.

intact model, and partial annulus fibers were removed in the building process of the ATd-ECD model. At the V-point of the C5-C6 left FJ, we built a $7 \mathrm{~mm}$ diameter tunnel from inside to outside, and partial capsular ligament and cartilage were removed to simulate posterior cervical foraminotomy in the PECF model. For the MACF model, we removed only the posterior part of UJs at $\mathrm{C} 5-\mathrm{C} 6$, without disc resection. For the ATc-ECD model, a $6 \mathrm{~mm}$ diameter tunnel was established from anterior-inferior towards posterior-superior of the C6 vertebral body; additionally, we did not interfere with the anterior longitudinal ligament and posterior longitudinal ligament during the process.

\section{Boundary and Loading Conditions}

A $1 \mathrm{Nm}$ moment was applied at the surface of the C3 superior endplate to imitate the movement of cervical spine flexion, extension, axial rotation, and lateral bending under a $50 \mathrm{~N}$ follower preload. The intermediate node of the endplate was coupled with each endplate surface, and these coupled nodes were connected by connector units (Cai et al., 2020b). The follower load was applied to each connector unit. The direction of the follower load was approximately tangential to the cervical spine physiological curve, and the follower load could provide a partial effect of muscle to the cervical movement (Bell et al., 2018). Hence, it could simulate physiological conditions better than the axial compressive load. We calculated the displacement of the intact model under moment and load during different motions; then, the displacement load was applied to every surgical model. The surface of the C7 inferior endplate is always immobilized completely when the cervical spine is in motion.

\section{RESULTS}

\section{Model Validation}

The loading conditions of model validation were identical with the previous experiments. The ROM of segments of the C3-C7 finite element model was calculated under the 

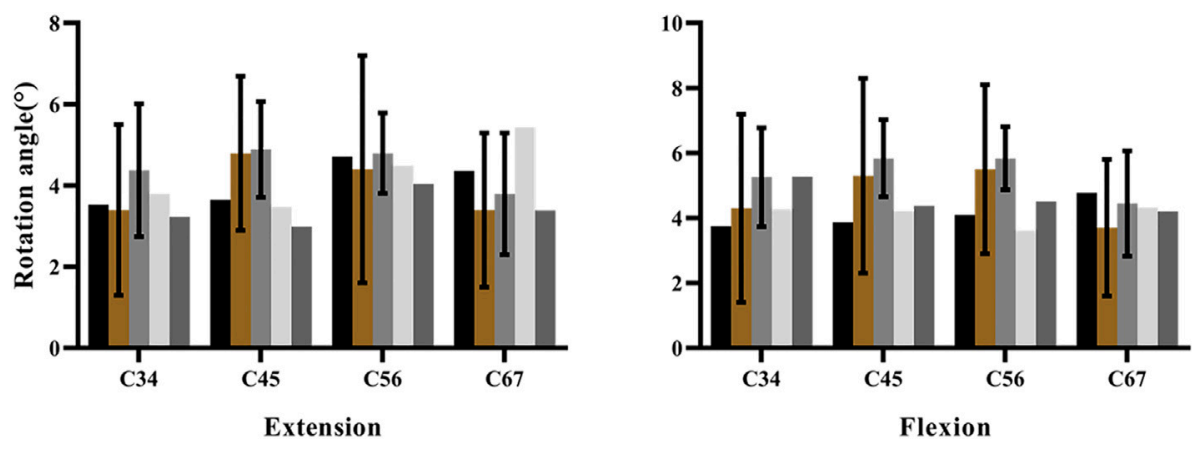

- Present study

Panjabi et al 2001

Liu et al 2016

Lee et al 2016

Cai et al 2020

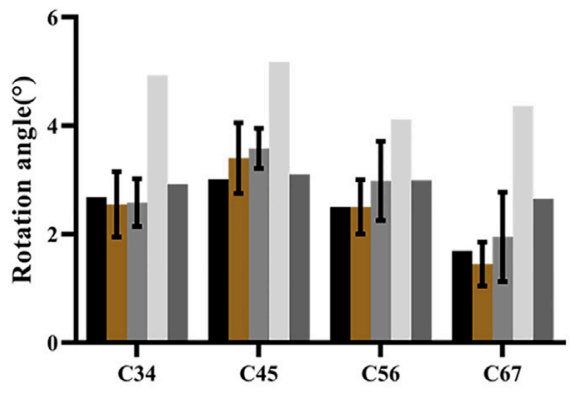

Axial rotation

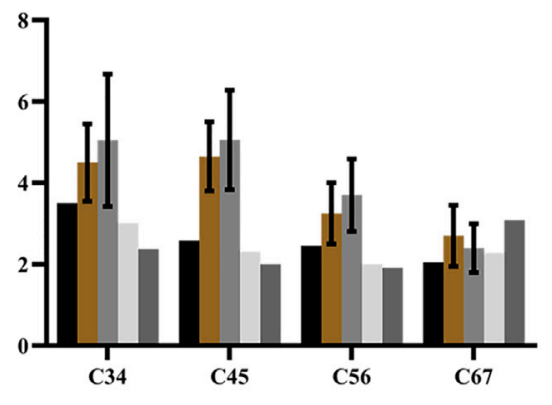

Lateral bending

FIGURE 3 | Validation of the intact C3-C7 finite element model.
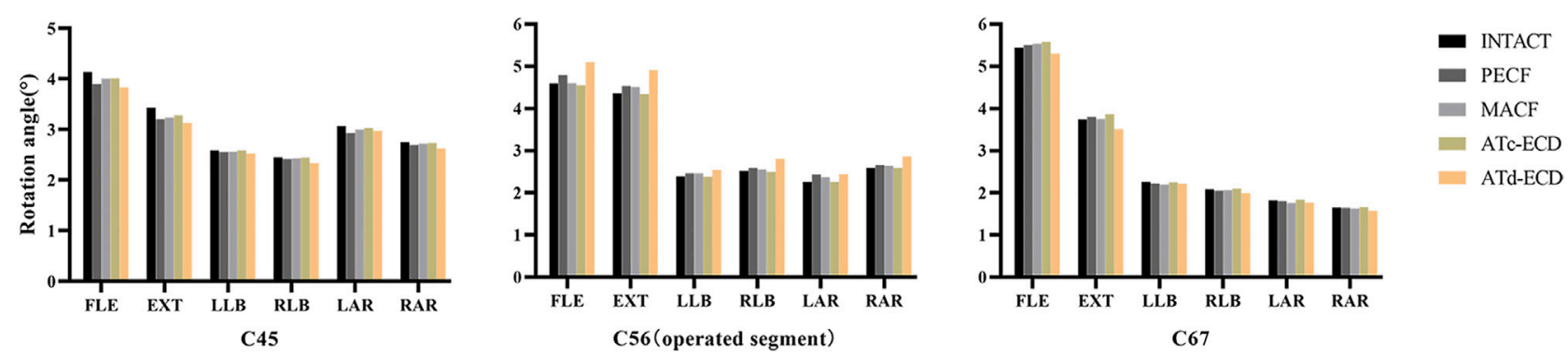

FIGURE 4 | The ROM of C4-C5, C5-C6, and C6-C7 in the intact model and surgical models. FLE, flexion; EXT, extension; LLB, left lateral bending; RLB, right lateral bending; LAR, left axial rotation; RAR, right axial rotation.

condition of $1 \mathrm{Nm}$ pure moment and compared with published data. The predicted ROM was consistent with in vitro experiments and previous finite element results (Panjabi et al., 2001; Lee et al., 2016; Liu et al., 2016; Cai et al., 2020a). The comparison between our results and previous ones is shown in Figure 3.

\section{ROM}

The ROM of operated and adjacent segments is shown in Figure 4. The most significant increase of the operated segment ROM occurred in the ATd-ECD, and the ROM increased by $11.07 \%, 12.77 \%, 11.56 \%$, and $10.51 \%$ during flexion, extension, right lateral bending, and right axial rotation, respectively. For the PECF model, we found that the ROM of the operated segment increased obviously during extension-flexion and axial rotation by $8.46 \%$ and $10.74 \%$, respectively. For the MACF model, axial rotation and lateral bending generated more mobility in the operated segment, followed by extension motion, and the percentage change was $6.93 \%, 4.57 \%$, and $3.56 \%$, respectively. However, the ROM of the operated segment in ATc-ECD showed a tiny decrease. For adjacent segments, the ROM of four surgical models showed a decreasing trend compared with the intact model.

\section{AFP and IDP}

The contour plots of the intervertebral disc pressure at C5-C6 are shown in Figure 5, and the IDP and AFP variations of different 


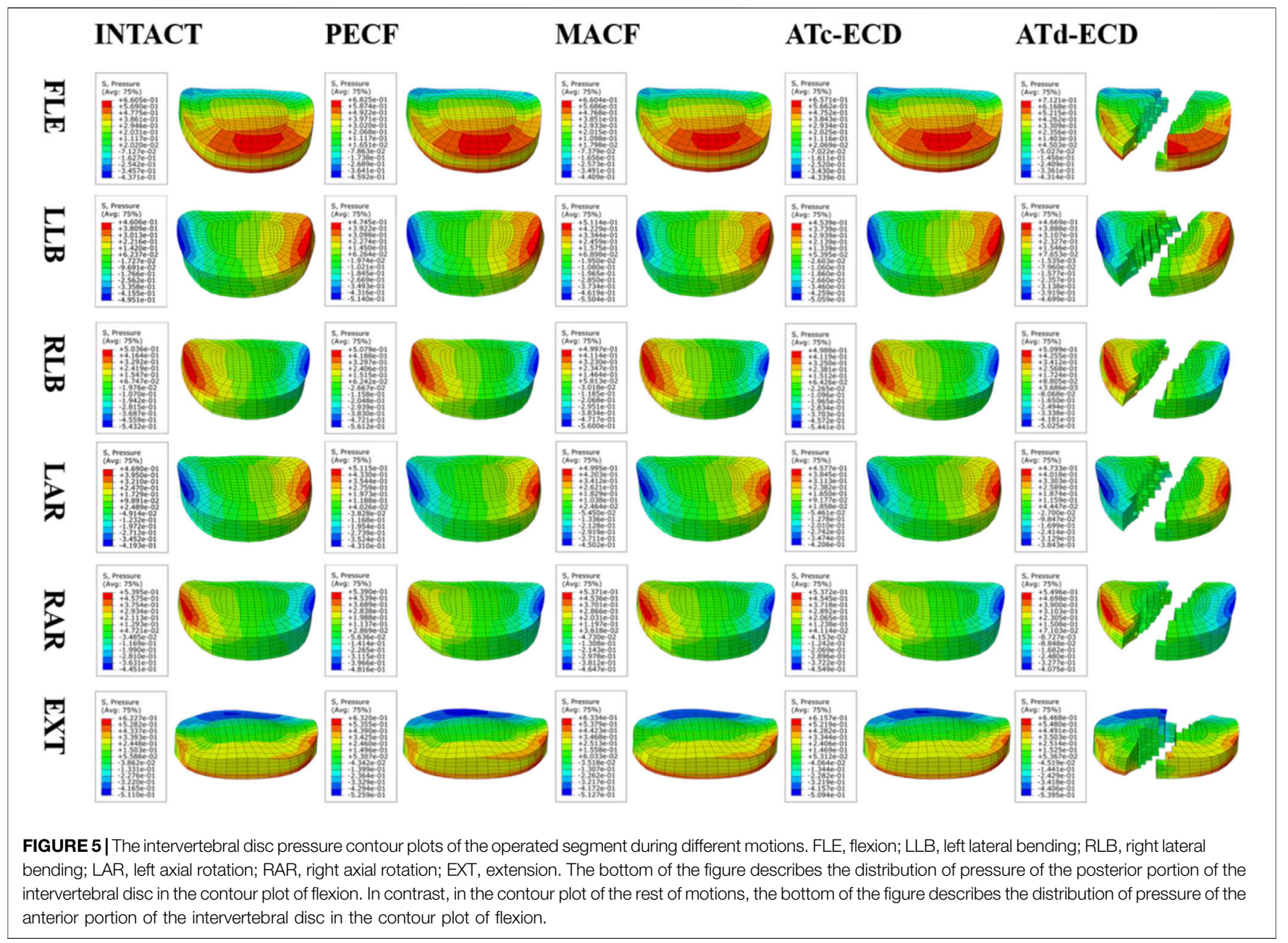

models are shown in Figure 6. In comparison with the other models, the IDP of the operated segment significantly increased in the MACF model, and the IDP increased by $13.95 \%, 9.75 \%$, and $9.19 \%$ during extension, left lateral bending, and left axial rotation, respectively. For the PECF model, the IDP of the operated segment increased obviously during left axial rotation, and the increase rate was $4.96 \%$. On the contrary, in the ATc-ECD and ATd-ECD models, the IDP of the operated segment decreased during different motions. The IDP of the operated segment decreased by $34.01 \%, 32.07 \%, 27.19 \%, 31.51 \%$, and $28.32 \%$ during flexion, left lateral bending, right lateral bending, left axial rotation, and right axial rotation, respectively, in the ATd-ECD model; on the other hand, in the ATc-ECD model, the maximum percentage of decrease was only $2.09 \%$ during extension. The variation trend of the AFP was similar with the IDP. Different from the IDP variation, in the ATd-ECD model, the AFP of the operated segment increased in different motions, and the maximum increase rate was $7.81 \%$ during flexion motion. For the PECF model, the AFP of the operated segment increased significantly during left axial rotation by $9.06 \%$. For the MACF model, the AFP of the operated segment increased significantly during left lateral bending by
$11.03 \%$. However, the AFP and the IDP decreased in the adjacent segments.

\section{FJs and UJs CPRESS}

The FJs and UJs CPRESS variations of different models are shown in Figure 7. According to the results, the increase of the operated segment FJs CPRESS in the ATd-ECD was apparent compared with the other models under different motions. For the ATc-ECD model, the variation of FJs CPRESS was inconspicuous. For all models, the PECF generated the maximum operated segment FJs CPRESS during right axial rotation, with a percentage increase of $16.77 \%$. The results suggest that different surgical models had lower effect on UJs. During lateral bending and axial rotation, the ipsilateral UJs CPRESS of the operated segment increased significantly when the cervical spine moved towards the surgical side in the MACF model, and the percentages were $12.24 \%$ and $8.82 \%$, respectively, whereas the left UJs CPRESS decreased by $53.47 \%$ during extension. For the PECF model, the UJs CPRESS of the operated segment increased by $9.48 \%$ during rotation. The changes of FJs and UJs CPRESS in adjacent segments were similar with the ones in ROM and IDP. 

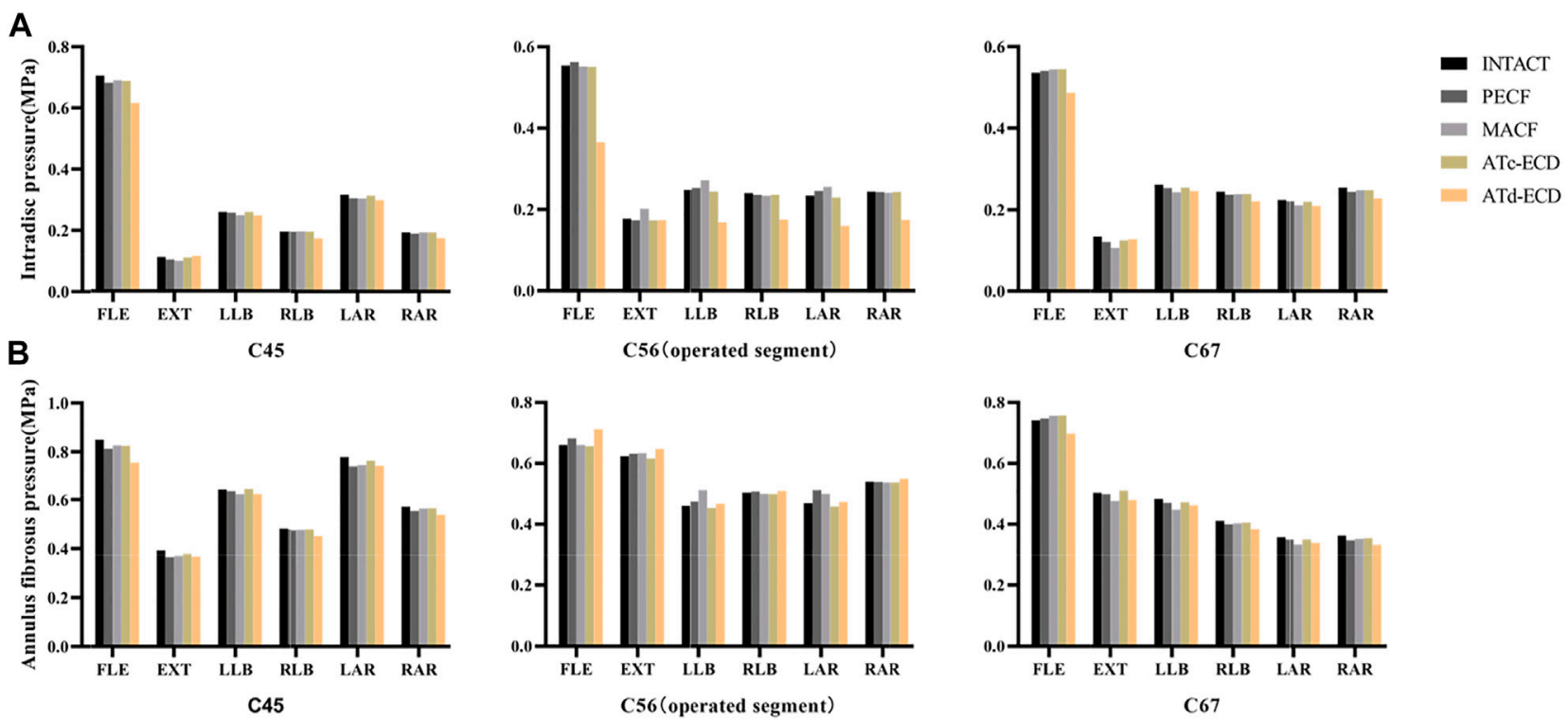

FIGURE 6 | The AFP and IDP of C4-C5, C5-C6, and C6-C7 in the intact model and surgical models. (A) Intradiscal pressure. (B) Annulus fibrosus pressure. FLE, flexion; EXT, extension; LLB, left lateral bending; RLB, right lateral bending; LAR, left axial rotation; RAR, right axial rotation.

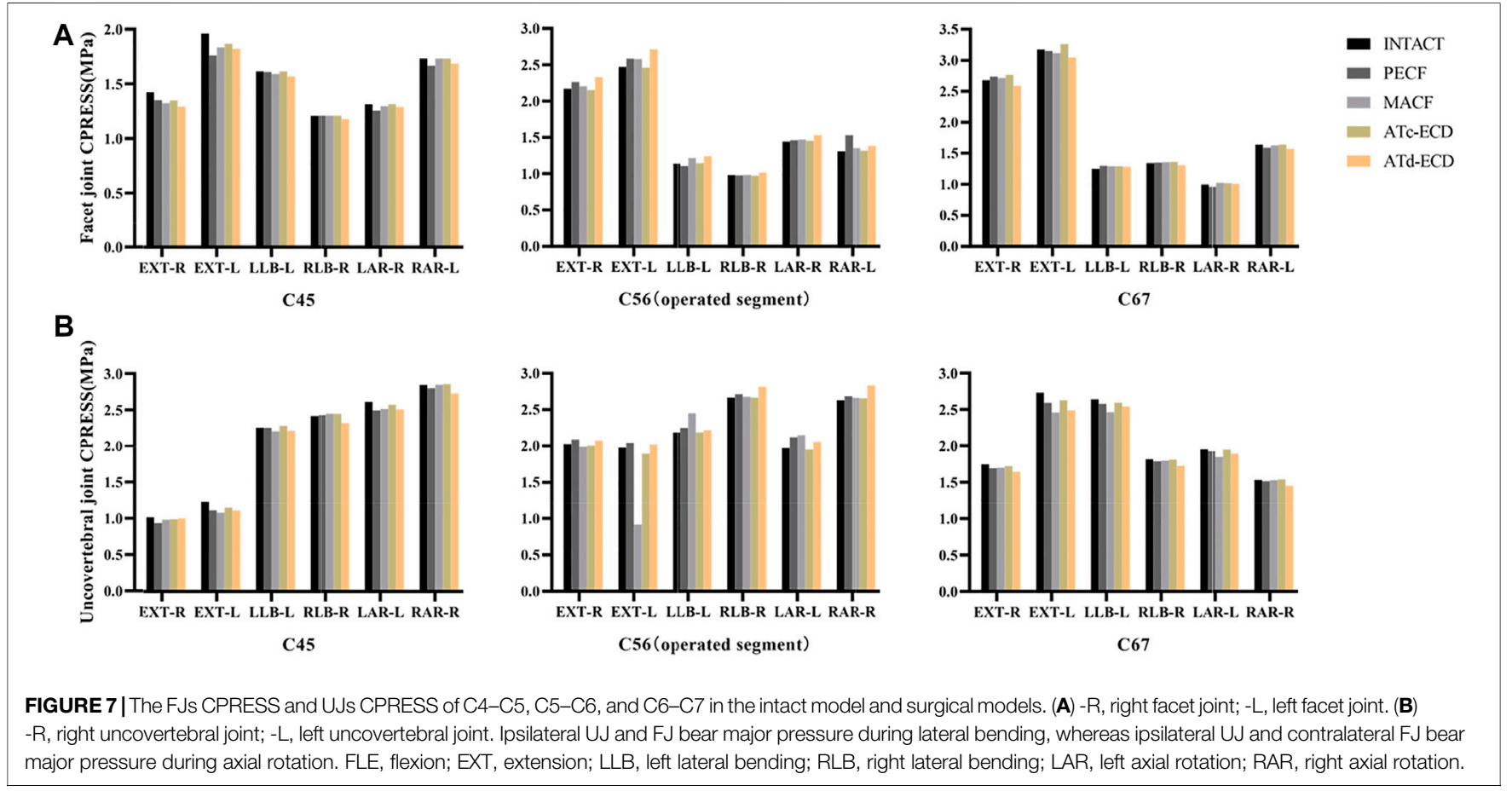

\section{DISCUSSION}

This study uses an indirectly validated three-dimensional finite element model of a normal C3-C7 segment to simulate cervical minimally invasive surgeries, including PECF, MACF, ATc-ECD, and ATd-ECD. Through comparison and analysis of the biomechanical changes of the four minimally invasive surgeries models, we hope to provide some evidences for surgeons to support their selection of appropriate surgical plans. From previous research (Ren et al., 2019; Yuchi et al., 2019; Chen et al., 2020; Ke et al., 2020), the assessment of biomechanics includes the following parameters: ROM, IDP, AFP, and FJs CPRESS. However, the biomechanical characteristics of UJs, which are a unique structure of the cervical spine, are not completely clear after minimally invasive 
surgeries. Therefore, in our study, we constructed the structure of UJs in the finite element model and calculated the contact pressure of UJs in the postoperative condition.

With regard to the ROM, the ATc-ECD model was closest to the intact model. Umebayashi et al. (2013) calculated the ROM of the functional spinal unit in the ATc-ECD using radiography measurements; the values were $5.0 \pm 2.7^{\circ}$ and $4.2 \pm 3.9^{\circ}$ in preoperative and postoperative conditions, respectively, and there was no significant difference for the ROM of the functional spinal unit between the two conditions. A similar situation occurred in our ATc-ECD model, whereas the ATd-ECD model significantly increased the segmental ROM compared with the other models, which was in accordance with existing studies (Yuchi et al., 2019; Chen et al., 2020). For the PECF model, part of the left-side FJ was removed at the C5-C6 segment, and FJs play an important role in limiting rotation and extension-flexion motion (Cai et al., 2020b). Similarly, the PECF significantly increased the operated segment ROM during rotation and extension-flexion and was lacking stability compared to the MACF model in our study. For the MACF model, the posterior part of the left UJ was resected. UJs are regarded as a stabilizer to limit cervical posterior translation and lateral bending; however, the function is undefined in the lower cervical spine, and there is a hypothesis that UJs provide more stability in rotation (Penning and Wilmink, 1987). Clausen et al. (1997) reported that the ROM on the C5-C6 segment increased by $25 \%$ and $14 \%$ during torsion and lateral bending, respectively, after UJ resection. Our study also supported this hypothesis by removing the posterior part of the left UJs in the MACF model. This is probably because of the greater disc facet angle in the lower cervical spine and UJs may permit more cervical torsion movement (Milne, 1991). Due to the surgical models and the intact model moving with the same displacement, the ROM of adjacent segments performed a compensatory decrease, which may be related to the increase of the operated segment (Chen et al., 2020). Hence, judging from the biomechanical viewpoint, the risk of adjacent segment degeneration might greatly decrease compared with anterior cervical discectomy and fusion after minimally invasive surgeries.

With regard to IDP and AFP, the MACF significantly increased, followed by the PECF model. We speculated that the UJs, which are load-bearing structures, might have a close relationship with the intervertebral disc. After removal of the UJs, there is a shift of a more compressive load from UJs to the neighboring intervertebral disc. Besides, an equilibrium mechanism may exit in the MACF and PECF. Rotation or lateral bending towards the direction of the surgical side caused IDP and AFP to increase, and the IDP and AFP decreased when the cervical spine shifted towards the opposite direction. Our results suggest that the ATc-ECD caused a very small intradiscal pressure decrease. However, the IDP decreased significantly after conducting the ATd-ECD, which was consistent with the previous research of Sun and Chen (Chen et al., 2020; Sun et al., 2021), and the situation of the AFP increase was consistent with the study by Yuchi et al. (2019). With the decrease of IDP, the capacity of the nucleus pulposus of supporting the load decreased. Afterwards the compressive load is transferred to the annulus fibrosus, and the height of the intervertebral disc will not be maintained (Sun et al., 2021).
For that reason, the height of the intervertebral disc decreased in previous clinical studies after performing the ATd-ECD.

For the FJs CPRESS, the model of the four minimally invasive surgeries showed an increase of a different magnitude in the operated segment, and the ATd-ECD model obtained maximum CPRESS. The increase of the CPRESS might cause joint abrasion and accelerate joint degeneration (Yuchi et al., 2019). When the PECF occurred in extension and rotation motion, the CPRESS of FJs increased significantly but decreased in lateral bending; this result is inconsistent with the study by Yuchi et al. (2019). This could be because of the ROM and contact area of FJs increasing during rotation and extension. Except for the ATc-ECD, the rest of the models may cause UJ degeneration at the operated segment, especially in rotation and lateral bending motions. However, the UJs CPRESS of the surgical side in the MACF decreased considerably during extension. We consider that the posterior part of UJs contributes importantly to stability (Kotani et al., 1998) and that it is the concentration area of pressure during extension. Therefore, the contact pressure showed an obvious decrease when removing the posterior part of UJs.

Furthermore, the ATd-ECD may result in irreversible iatrogenic disc injury and poorer cervical stability; still, Sun et al. (2021) reported that cervical stability was affected by the approach of angle and surgical diameter when conducting the ATd-ECD. In their study, cervical stability changed minimally when the angle of approach was $90^{\circ}$. For building a targeted channel and reducing disc injury as much as possible, surgeons often need a contrast agent mixed with methylenum coeruleum; nevertheless, the stain or contrast agent may bring some potential risk, such as toxic effect and discitis (Yang et al., 2014). Meanwhile, the intervertebral disc height cannot be lower than the endoscope diameter, which is currently $3-4 \mathrm{~mm}$; thus, an excessively low disc height is a restriction (Ahn, 2016; Ren et al., 2020). The ATc-ECD and ATd-ECD are similar in technology. Previous studies have demonstrated that the ATc-ECD has hardly any impact on vertebrae strength if surgeons choose an endoscopic system with a diameter within $6 \mathrm{~mm}$ and a lateral approach (Umebayashi et al., 2013; Wu et al., 2018). Also, Ren et al. (2020) reported that bone defect within $6 \mathrm{~mm}$ could heal after the ATc-ECD, and the complete healing rate reached up to $94.28 \%$. Therefore, in this study, we focus more on segmental stability rather than bone strength.

Our results show that the MACF can maintain segmental stability well, but there are some setbacks to its use. Considering that the vertebral artery is closely located at the UJ lateral side, vertebral artery injury could be a serious intraoperative complication when performing the MACF (Saringer et al., 2002). In addition, the distance between the lateral wall of the uncinate process and artery is disparate at different segments. Kim et al. (2012) reported that the shortest distance is located at the $\mathrm{C} 3-\mathrm{C} 4$ segment, and the farthest distance is located at the $\mathrm{C} 6-\mathrm{C} 7$ segment. This means that the MACF procedure cannot be carried out at each segment, and surgeons need to prudentially estimate the positional relationship between UJs and vertebral artery before operation. Meanwhile, it is noteworthy that the gap between UJs and vertebral artery displays a constriction when UJ hypertrophy or serious disc degeneration occurs (Urbanschitz et al., 2021). Hence, the risk of vertebral artery injury may further increase.

The spine is formed from complex and interrelated structures containing the vertebral body, FJ, intervertebral 
disc, ligaments, and muscle tissue, which together contribute to spine stability and transmission of force, and one structure degeneration or injury will affect the rest of the structures (Kumaresan et al., 2001; O'Leary et al., 2018; Vergroesen et al., 2015). In the present study, the standard model was constructed on the basis of a healthy cervical spine rather than a degenerated cervical spine. A degenerated spine has a decreased segmental motion and intradiscal pressure and increased FJ load compared with a healthy spine (Hussain et al., 2010; Vergroesen et al., 2015; Cai et al., 2020a). Hence, there are differences between healthy and pathological postoperation models in the calculated parameters, including ROM, CPRESS, and intervertebral disc pressure. And it will cause some deviations such that finite element analysis results completely substitute for a variety of pathologic postoperative outcomes. However, the morphology of a pathological cervical spine is diverse, including different cervical curvatures and intervertebral disc height loss or not, which causes the diversification of biomechanical results after operation. In addition, the indications of different minimally invasive surgeries are not all the same, which means there are restrictions in choosing an appropriate pathological model. Though lacking individual specificity, CT data of a healthy male contribute to the unification of variables, and the study results are still universal and adaptable. Our results might be closer to some clinical cases that suffer from pure cervical disc herniation without apparent structure change of the cervical spine.

There are several limitations in our study that should be reported. Firstly, the validation method of the standard model is an indirect way of comparison with published data. Although most finite element analyses of the spine used indirect validation (Jones and Wilcox, 2008), the accuracy and precision of finite element results decreased because of unclear in vitro experiment conditions, large standard deviation, and lack of specific material properties. Thus, the calculated pressures of the intervertebral disc and articular cartilage are not equal to the actual value. Combined with identical data of in vitro experiments, developing a specific finite element model might be a trend. Secondly, although the follow load could provide a part effect of muscle to the cervical spine, the detailed and complicated function of muscle-tospine movement cannot be simulated. Thirdly, there is a lack of recognized method of constructing UJs. We simplified UJs in the model, which might lead to loss of some details. Fourthly, for good convergence in the calculating process, we chose homogeneous and linear materials. Although they have partial impact on the localized mechanical environment, they do not affect the cervical spine kinetics. The material properties need to be noticed if the direction of the study changes. Overall, our predicted results might not represent the precise clinical numerical value, but they could predict a dependable variation tendency during postoperative cervical surgery in different motions. For obtaining comprehensive and accurate biomechanical data, more in vivo and in vitro experiments should be conducted in the future.

\section{CONCLUSION}

In our study, all types of minimally invasive surgeries displayed good biomechanical stability. From the standpoint of biomechanics, the physiological status of the ATc-ECD was close to the normal cervical model, followed by the MACF model. The ATd-ECD model might significantly accelerate disc and joint degeneration compared with the other three types of minimally invasive surgeries. Considering safety and conditions of application, the PECF and ATc-ECD may be relatively suitable techniques for cervical spondylotic radiculopathy. Certainly, surgeons can choose the appropriate procedures according to their proficiency level and radiological results, such as intervertebral disc height and types of disc herniation.

\section{DATA AVAILABILITY STATEMENT}

The original contributions presented in the study are included in the article/Supplementary Material, further inquiries can be directed to the corresponding author.

\section{ETHICS STATEMENT}

This study was approved by the ethics committee of The Second Hospital of Jilin University (No. 122), and informed consent was obtained from volunteers.

\section{AUTHOR CONTRIBUTIONS}

1) The conception and design of the study and the analysis and interpretation of data: TH. 2) The acquisition of data and the analysis and interpretation of data: JZ, TY, and JW. 3) Drafting the article or revising it critically for important intellectual content: TY, RL, ZY, HD, JA, LQ, XN, and WX. 4) Final approval of the version to be submitted: QL.

\section{FUNDING}

This study was financially supported by the program of Scientific Development of Jilin Province (20190201066JC), the Special Health Project of Jilin Province Department of Finance (2020SC2T041), the Health Appropriate Technology Poverty Alleviation Project of Jilin Province (2018FP043), and Science and Technology Department of Jilin Province (20210101265JC).

\section{ACKNOWLEDGMENTS}

We would like to thank Elsevier (webshop.elsevier.com) for language editing services. 


\section{REFERENCES}

Ahn, Y. (2016). Percutaneous Endoscopic Cervical Discectomy Using Working Channel Endoscopes. Expert Rev. Med. Devices 13, 601-610. doi:10.1080/ 17434440.2016.1180245

Ahn, Y. (2020). The Current State of Cervical Endoscopic Spine Surgery: an Updated Literature Review and Technical Considerations. Expert Rev. Med. Devices 17, 1285-1292. doi:10.1080/17434440.2020.1853523

Ames, C. P., Acosta, F. L., Jr., Chi, J., Iyengar, J., Muiru, W., Acaroglu, E., et al. (2005). Biomechanical Comparison of Posterior Lumbar Interbody Fusion and Transforaminal Lumbar Interbody Fusion Performed at 1 and 2 Levels. Spine 30, E562-E566. doi:10.1097/01.brs.0000180505.80347.b1

Bell, K. M., Yan, Y., Hartman, R. A., and Lee, J. Y. (2018). Influence of Follower Load Application on Moment-Rotation Parameters and Intradiscal Pressure in the Cervical Spine. J. Biomech. 76, 167-172. doi:10.1016/j.jbiomech.2018.05.031

Boreadis, A. G., and Gershon-Cohen, J. (1956). Luschka Joints of the Cervical Spine. Radiology 66, 181-187. doi:10.1148/66.2.181

Brismée, J.-M., Sizer, P. S., Jr., Dedrick, G. S., Sawyer, B. G., and Smith, M. P. (2009). Immunohistochemical and Histological Study of Human Uncovertebral Joints. Spine 34, 1257-1263. doi:10.1097/BRS.0b013e31819b2b5d

Cai, X.-Y., Sang, D., Yuchi, C.-X., Cui, W., Zhang, C., Du, C.-F., et al. (2020a). Using Finite Element Analysis to Determine Effects of the Motion Loading Method on Facet Joint Forces after Cervical Disc Degeneration. Comput. Biol. Med. 116, 103519. doi:10.1016/j.compbiomed.2019.103519

Cai, X.-Y., Yuchi, C.-X., Du, C.-F., and Mo, Z.-J. (2020b). The Effect of Follower Load on the Range of Motion, Facet Joint Force, and Intradiscal Pressure of the Cervical Spine: a Finite Element Study. Med. Biol. Eng. Comput. 58, 1695-1705. doi:10.1007/s11517-020-02189-7

Chen, C., Yuchi, C.-X., Gao, Z., Ma, X., Zhao, D., Li, J.-W., et al. (2020). Comparative Analysis of the Biomechanics of the Adjacent Segments after Minimally Invasive Cervical Surgeries versus Anterior Cervical Discectomy and Fusion: A Finite Element Study. J. Orthopaedic Translation 23, 107-112. doi:10.1016/j.jot.2020.03.006

Chen, X., Gao, J. A., Du, Q., Qiao, Y., Kong, W. J., and Liao, W. B. (2021). Percutaneous Full-Endoscopic Anterior Transcorporeal Cervical Discectomy for the Treatment of Cervical Disc Herniation: Surgical Design and Results. Pain physician 24, E811-e819.

Choi, G., Lee, S.-H., Bhanot, A., Chae, Y. S., Jung, B., and Lee, S. (2007). Modified Transcorporeal Anterior Cervical Microforaminotomy for Cervical Radiculopathy: a Technical Note and Early Results. Eur. Spine J. 16, 1387-1393. doi:10.1007/s00586-006-0286-6

Clausen, J. D., Goel, V. K., Traynelis, V. C., and Scifert, J. (1997). Uncinate Processes and Luschka Joints Influence the Biomechanics of the Cervical Spine: Quantification Using a Finite Element Model of the C5-C6 Segment. J. Orthop. Res. 15, 342-347. doi:10.1002/jor.1100150305

Clifton, W., Valero-Moreno, F., Vlasak, A., Damon, A., Tubbs, R. S., Merrill, S., et al. (2020). Microanatomical Considerations for Safe Uncinate Removal during Anterior Cervical Discectomy and Fusion: 10-year Experience. Clin. Anat. 33, 920-926. doi:10.1002/ca.23596

Clifton, W., Williams, D., and Pichelmann, M. (2019). How I Do it: Total Uncinatectomy during Anterior Diskectomy and Fusion for Cervical Radiculopathy Caused by Uncovertebral Joint Hypertrophy. Acta Neurochir 161, 2229-2232. doi:10.1007/s00701-019-04033-w

Deng, Z.-L., Chu, L., Chen, L., and Yang, J.-S. (2016). Anterior Transcorporeal Approach of Percutaneous Endoscopic Cervical Discectomy for Disc Herniation at the C4-C5 Levels: a Technical Note. Spine J. 16, 659-666. doi:10.1016/j.spinee.2016.01.187

Guan, T., Zhang, Y., Anwar, A., Zhang, Y., and Wang, L. (2020). Determination of Three-Dimensional Corrective Force in Adolescent Idiopathic Scoliosis and Biomechanical Finite Element Analysis. Front. Bioeng. Biotechnol. 8, 963. doi:10.3389/fbioe.2020.00963

Haijun, M., Haoping, Z., Honggang, Z., and Xiaobing, Z. (2021). Clinical Study on the Efficacy and Safety of Percutaneous Endoscopic Anterior Cervical Discectomy in the Treatment of Cervical Disc Herniation. Int. Orthopaedics (Sicot) 45, 1247-1256. doi:10.1007/s00264-021-04982-x

Hua, W., Zhi, J., Ke, W., Wang, B., Yang, S., Li, L., et al. (2020). Adjacent Segment Biomechanical Changes after One- or Two-Level Anterior Cervical Discectomy and
Fusion Using Either a Zero-Profile Device or Cage Plus Plate: A Finite Element Analysis. Comput. Biol. Med. 120, 103760. doi:10.1016/j.compbiomed.2020.103760

Hussain, M., Natarajan, R. N., An, H. S., and Andersson, G. B. J. (2010). Motion Changes in Adjacent Segments Due to Moderate and Severe Degeneration in C5-C6 Disc. Spine 35, 939-947. doi:10.1097/BRS.0b013e3181bd419b

Jho, H.-D. (1997). Decompression via Microsurgical Anterior Foraminotomy for Cervical Spondylotic Myelopathy. J. Neurosurg. 86, 297-302. doi:10.3171/ jns.1997.86.2.0297

Jones, A. C., and Wilcox, R. K. (2008). Finite Element Analysis of the Spine: towards a Framework of Verification, Validation and Sensitivity Analysis. Med. Eng. Phys. 30, 1287-1304. doi:10.1016/j.medengphy.2008.09.006

Ke, W., Zhi, J., Hua, W., Wang, B., Lu, S., Fan, L., et al. (2020). Percutaneous Posterior Full-Endoscopic Cervical Foraminotomy and Discectomy: a Finite Element Analysis and Radiological Assessment. Comp. Methods Biomech. Biomed. Eng. 23, 805-814. doi:10.1080/10255842.2020.1765162

Kim, C. H., Kim, K.-T., Chung, C. K., Park, S. B., Yang, S. H., Kim, S. M., et al. (2015). Minimally Invasive Cervical Foraminotomy and Diskectomy for Laterally Located Soft Disk Herniation. Eur. Spine J. 24, 3005-3012. doi:10.1007/s00586-015-4198-1

Kim, J.-S., Eun, S. S., Prada, N., Choi, G., and Lee, S.-H. (2011). Modified Transcorporeal Anterior Cervical Microforaminotomy Assisted by O-Arm-Based Navigation: a Technical Case Report. Eur. Spine J. 20 (2), 147-152. doi:10.1007/s00586-010-1454-2

Kim, S.-H., Lee, J. H., Kim, J. H., Chun, K. S., Doh, J. W., and Chang, J. C. (2012). Anatomical Morphometric Study of the Cervical Uncinate Process and Surrounding Structures. J. Korean Neurosurg. Soc. 52, 300-305. doi:10.3340/jkns.2012.52.4.300

Kotani, Y., Mcnulty, P. S., Abumi, K., Cunningham, B. W., Kaneda, K., and Mcafee, P. C. (1998). The Role of Anteromedial Foraminotomy and the Uncovertebral Joints in the Stability of the Cervical Spine. Spine 23, 1559-1565. doi:10.1097/00007632-199807150-00011

Kotil, K., and Bilge, T. (2008). Prospective Study of Anterior Cervical Microforaminotomy for Cervical Radiculopathy. J. Clin. Neurosci. 15, 749-756. doi:10.1016/j.jocn.2007.04.013

Kumaresan, S., Yoganandan, N., Pintar, F. A., Maiman, D. J., and Goel, V. K. (2001). Contribution of Disc Degeneration to Osteophyte Formation in the Cervical Spine: a Biomechanical Investigation. J. Orthop. Res. 19, 977-984. doi:10.1016/s0736-0266(01)00010-9

Lee, D.-H., Cho, J. H., Baik, J.-M., Joo, Y.-S., Park, S., Min, W.-K., et al. (2018). Does Additional Uncinate Resection Increase Pseudarthrosis Following Anterior Cervical Discectomy and Fusion? Spine (Phila $\mathrm{Pa}$ 1976) 43, 97-104. doi:10.1097/BRS.0000000000002271

Lee, J. H., and Lee, S.-H. (2014). Clinical and Radiographic Changes after Percutaneous Endoscopic Cervical Discectomy: a Long-Term Follow-Up. Photomed. Laser Surg. 32, 663-668. doi:10.1089/pho.2014.3806

Lee, J. H., Park, W. M., Kim, Y. H., and Jahng, T.-A. (2016). A Biomechanical Analysis of an Artificial Disc with a Shock-Absorbing Core Property by Using Whole-Cervical Spine Finite Element Analysis. Photomed. Laser Surg. Spine 41, E893-E901. doi:10.1097/BRS.0000000000001468

Lee, S.-H., Im, Y.-J., Kim, K.-T., Kim, Y.-H., Park, W.-M., and Kim, K. (2011). Comparison of Cervical Spine Biomechanics after Fixed- and Mobile-Core Artificial Disc Replacement. Spine 36, 700-708. doi:10.1097/BRS.0b013e3181f5cb87

Liu, Q., Guo, Q., Yang, J., Zhang, P., Xu, T., Cheng, X., et al. (2016). Subaxial Cervical Intradiscal Pressure and Segmental Kinematics Following Atlantoaxial Fixation in Different Angles. World Neurosurg. 87, 521-528. doi:10.1016/j.wneu.2015.09.025

Maduri, R., Bobinski, L., and Duff, J. M. (2020). Minimally Invasive Anterior Foraminotomy for Cervical Radiculopathy: How I Do it. Acta Neurochir 162, 679-683. doi:10.1007/s00701-019-04201-y

Milne, N. (1991). The Role of Zygapophysial Joint Orientation and Uncinate Processes in Controlling Motion in the Cervical Spine. J. Anat. 178, 189-201.

Mo, Z., Zhao, Y., Du, C., Sun, Y., Zhang, M., and Fan, Y. (2015). Does Location of Rotation center in Artificial Disc Affect Cervical Biomechanics? Spine 40, E469-E475. doi:10.1097/BRS.0000000000000818

Noh, S. H., Park, J. Y., Kuh, S. U., Chin, D. K., Kim, K. S., Cho, Y. E., et al. (2020). Association of Complete Uncinate Process Removal on 2-year Assessment of Radiologic Outcomes: Subsidence and Sagittal Balance in Patients Receiving One-Level Anterior Cervical Discectomy and Fusion. BMC Musculoskelet. Disord. 21, 439. doi:10.1186/s12891-020-03443-7 
O'leary, S. A., Paschos, N. K., Link, J. M., Klineberg, E. O., Hu, J. C., and Athanasiou, K. A. (2018). Facet Joints of the Spine: Structure-Function Relationships, Problems and Treatments, and the Potential for Regeneration. Annu. Rev. Biomed. Eng. 20, 145-170. doi:10.1146/annurevbioeng-062117-120924

Panjabi, M. M., Crisco, J. J., Vasavada, A., Oda, T., Cholewicki, J., Nibu, K., et al. (2001). Mechanical Properties of the Human Cervical Spine as Shown by Three-Dimensional Load-Displacement Curves. Spine 26, 2692-2700. doi:10.1097/00007632-200112150-00012

Penning, L., and Wilmink, J. T. (1987). Rotation of the Cervical Spine. Spine 12, 732-738. doi:10.1097/00007632-198710000-00003

Quillo-Olvera, J., Lin, G.-X., and Kim, J.-S. (2018). Percutaneous Endoscopic Cervical Discectomy: a Technical Review. Ann. Transl. Med. 6, 100. doi:10.21037/atm.2018.02.09

Ren, J., Li, R., Zhu, K., Han, X., Liu, X., He, Y., et al. (2019). Biomechanical Comparison of Percutaneous Posterior Endoscopic Cervical Discectomy and Anterior Cervical Decompression and Fusion on the Treatment of Cervical Spondylotic Radiculopathy. J. Orthop. Surg. Res. 14, 71. doi:10.1186/s13018-019-1113-1

Ren, Y., Yang, J., Chen, C.-M., Liu, K., Wang, X.-F., Wei, J.-M., et al. (2020). Outcomes of Discectomy by Using Full-Endoscopic Visualization Technique via the Transcorporeal and Transdiscal Approaches in the Treatment of Cervical Intervertebral Disc Herniation: A Comparative Study. Biomed. Res. Int. 2020, 1-7. doi:10.1155/2020/5613459

Ruetten, S., Komp, M., Merk, H., and Godolias, G. (2009). Full-endoscopic Anterior Decompression versus Conventional Anterior Decompression and Fusion in Cervical Disc Herniations. Int. Orthopaedics (Sicot) 33, 1677-1682. doi:10.1007/s00264-008-0684-y

Ruetten, S., Komp, M., Merk, H., and Godolias, G. (2008). Full-Endoscopic Cervical Posterior Foraminotomy for the Operation of Lateral Disc Herniations Using 5.9-mm Endoscopes. Spine 33, 940-948. doi:10.1097/ BRS.0b013e31816c8b67

Sang, D., Du, C.-F., Wu, B., Cai, X.-Y., Cui, W., Yuchi, C.-X., et al. (2021). The Effect of Cervical Intervertebral Disc Degeneration on the Motion Path of Instantaneous center of Rotation at Degenerated and Adjacent Segments: A Finite Element Analysis. Comput. Biol. Med. 134, 104426. doi:10.1016/ j.compbiomed.2021.104426

Saringer, W. F., Reddy, B., Nöbauer-Huhmann, I., Regatschnig, R., Reddy, M., Tschabitscher, M., et al. (2003). Endoscopic Anterior Cervical Foraminotomy for Unilateral Radiculopathy: Anatomical Morphometric Analysis and Preliminary Clinical Experience. J. Neurosurg. 98, 171-180. doi:10.3171/ spi.2003.98.2.0171

Saringer, W., Nöbauer, I., Reddy, M., Tschabitscher, M., and Horaczek, A. (2002). Microsurgical Anterior Cervical Foraminotomy (Uncoforaminotomy) for Unilateral Radiculopathy: Clinical Results of a New Technique. Acta Neurochir (Wien) 144, 685-694. doi:10.1007/s00701002-0953-2

Snyder, J. T., Tzermiadianos, M. N., Ghanayem, A. J., Voronov, L. I., Rinella, A., Dooris, A., et al. (2007). Effect of Uncovertebral Joint Excision on the Motion Response of the Cervical Spine after Total Disc Replacement. Spine 32, 2965-2969. doi:10.1097/BRS.0b013e31815cd482

Sun, M.-S., Yuchi, C.-X., Cai, X.-Y., Du, C.-F., and Mo, Z.-J. (2021). Parametric Study of Anterior Percutaneous Endoscopic Cervical Discectomy (APECD). Comp. Methods Biomech. Biomed. Eng. 24, 687-699. doi:10.1080/10255842.2020.1846186

Taşçioğlu, A. O., Attar, A., and Taşçioǧlu, B. (2001). Microsurgical Anterior Cervical Foraminotomy (Uncinatectomy) for Cervical Disc Herniation. J. Neurosurg. 94, 121-125. doi:10.3171/spi.2001.94.1.0121

Umebayashi, D., Hara, M., Nakajima, Y., Nishimura, Y., and Wakabayashi, T. (2013). Transvertebral Anterior Cervical Foraminotomy: Midterm Outcomes of Clinical and Radiological Assessments Including the Finite Element Method. Eur. Spine J. 22, 2884-2890. doi:10.1007/ s00586-013-2974-3

Urbanschitz, L., Merat, S., Bensler, S., Lenz, C. G., Mameghani, A. T., and Eid, K. (2021). Cervical Disc Degeneration Reduces Distance between Vertebral Artery and Surgical Landmarks. Clin. Neurol. Neurosurg. 200, 106332. doi:10.1016/ j.clineuro.2020.106332

Vergroesen, P.-P. A., Kingma, I., Emanuel, K. S., Hoogendoorn, R. J. W., Welting, T. J., Van Royen, B. J., et al. (2015). Mechanics and Biology in Intervertebral
Disc Degeneration: a Vicious circle. Osteoarthritis and Cartilage 23, 1057-1070. doi:10.1016/j.joca.2015.03.028

Wang, B., Ke, W., Hua, W., Zeng, X., and Yang, C. (2020). Biomechanical Evaluation and the Assisted 3D Printed Model in the Patient-specific Preoperative Planning for Thoracic Spinal Tuberculosis: A Finite Element Analysis. Front. Bioeng. Biotechnol. 8, 807. doi:10.3389/fbioe.2020.00807

Wang, Z., Zhao, H., Liu, J.-m., Tan, L.-w., Liu, P., and Zhao, J.-h. (2016). Resection or Degeneration of Uncovertebral Joints Altered the Segmental Kinematics and Load-Sharing Pattern of Subaxial Cervical Spine: A Biomechanical Investigation Using a C2-T1 Finite Element Model. J. Biomech. 49, 2854-2862. doi:10.1016/j.jbiomech.2016.06.027

Wo, J., Lv, Z., Wang, J., Shen, K., Zhu, H., Liu, Y., et al. (2021). Biomechanical Analysis of Cervical Artificial Disc Replacement Using Cervical Subtotal Discectomy Prosthesis. Front. Bioeng. Biotechnol. 9, 680769. doi:10.3389/ fbioe.2021.680769

Won, S., Kim, C. H., Chung, C. K., Choi, Y., Park, S. B., Moon, J. H., et al. (2017). Comparison of Cervical Sagittal Alignment and Kinematics after Posterior FullEndoscopic Cervical Foraminotomy and Discectomy According to Preoperative Cervical Alignment. Pain physician 20, 77-87.

Wong, C.-E., Hu, H.-T., Hsieh, M.-P., and Huang, K.-Y. (2020). Optimization of Three-Level Cervical Hybrid Surgery to Prevent Adjacent Segment Disease: A Finite Element Study. Front. Bioeng. Biotechnol. 8, 154. doi:10.3389/ fbioe.2020.00154

Wu, P. H., Kim, H. S., Lee, Y. J., Kim, D. H., Lee, J. H., Yang, K.-H., et al. (2021a). Posterior Endoscopic Cervical Foramiotomy and Discectomy: Clinical and Radiological Computer Tomography Evaluation on the Bony Effect of Decompression with 2 Years Follow-Up. Eur. Spine J. 30, 534-546. doi:10.1007/s00586-020-06637-8

Wu, T.-k., Meng, Y., Liu, H., Wang, B.-y., Hong, Y., Rong, X., et al. (2019). Biomechanical Effects on the Intermediate Segment of Noncontiguous Hybrid Surgery with Cervical Disc Arthroplasty and Anterior Cervical Discectomy and Fusion: a Finite Element Analysis. Spine J. 19, 1254-1263. doi:10.1016/ j.spinee.2019.02.004

Wu, W.-k., Yan, Z. J., Zhang, T.-f., Liao, C.-g., Liang, K.-l., Chen, L., et al. (2018). Biomechanical Influences of Transcorporeal Tunnels on C4 Vertebra under Physical Compressive Load under Flexion Movement: A Finite Element Analysis. World Neurosurg. 114, e199-e208. doi:10.1016/ j.wneu.2018.02.140

Wu, W., Han, Z., Hu, B., Du, C., Xing, Z., Zhang, C., et al. (2021b). A Graphical Guide for Constructing a Finite Element Model of the Cervical Spine with Digital Orthopedic Software. Ann. Transl Med. 9, 169. doi:10.21037/atm-20-2451

Yang, J.-S., Chu, L., Chen, L., Chen, F., Ke, Z.-Y., and Deng, Z.-L. (2014). Anterior or Posterior Approach of Full-Endoscopic Cervical Discectomy for Cervical Intervertebral Disc Herniation? A Comparative Cohort Study. Spine 39, 1743-1750. doi:10.1097/BRS.0000000000000508

Yuchi, C.-X., Sun, G., Chen, C., Liu, G., Zhao, D., Yang, H., et al. (2019). Comparison of the Biomechanical Changes after Percutaneous FullEndoscopic Anterior Cervical Discectomy versus Posterior Cervical Foraminotomy at C5-C6: A Finite Element-Based Study. World Neurosurg. 128, e905-e911. doi:10.1016/j.wneu.2019.05.025

Conflict of Interest: The authors declare that the research was conducted in the absence of any commercial or financial relationships that could be construed as a potential conflict of interest.

Publisher's Note: All claims expressed in this article are solely those of the authors and do not necessarily represent those of their affiliated organizations, or those of the publisher, the editors and the reviewers. Any product that may be evaluated in this article, or claim that may be made by its manufacturer, is not guaranteed or endorsed by the publisher.

Copyright (C) $2021 \mathrm{He}$, Zhang, Yu, Wu, Yuan, Liu, Yun, Du, Qi, An, Xue, Nie and Liu. This is an open-access article distributed under the terms of the Creative Commons Attribution License (CC BY). The use, distribution or reproduction in other forums is permitted, provided the original author(s) and the copyright owner(s) are credited and that the original publication in this journal is cited, in accordance with accepted academic practice. No use, distribution or reproduction is permitted which does not comply with these terms. 